Research Article

\title{
Chaotic Vibration and Comfort Analysis of Nonlinear Full-Vehicle Model Excited by Consecutive Speed Control Humps
}

\author{
Zhiyong Yang, ${ }^{1,2}$ Shan Liang, ${ }^{2}$ Qin Zhu, ${ }^{3}$ Tong Zhou, ${ }^{1}$ and Cheng Zeng ${ }^{4}$ \\ ${ }^{1}$ College of Information Engineering, Chongqing Institute of Engineering, Chongqing 402260, China \\ ${ }^{2}$ College of Automation, Chongqing University, Chongqing 400044, China \\ ${ }^{3}$ Department of Mechanical Engineering, Oyama National College of Technology, Oyama 323-0806, Japan \\ ${ }^{4}$ College of Science, Guizhou Institute of Technology, Guiyang 550003, China
}

Correspondence should be addressed to Zhiyong Yang; zy.yang@cqu.edu.cn

Received 13 July 2014; Revised 29 September 2014; Accepted 29 September 2014; Published 1 December 2014

Academic Editor: Yan-Jun Liu

Copyright (C) 2014 Zhiyong Yang et al. This is an open access article distributed under the Creative Commons Attribution License, which permits unrestricted use, distribution, and reproduction in any medium, provided the original work is properly cited.

\begin{abstract}
When vehicles are driven on consecutive speed control humps, the parameters of speed control humps such as height, width, and space and vehicle's speed are the important factors to affect safety and comfort of passengers. The paper assumes that the excitation function of the consecutive speed control humps is a half-sine wave and a SCHs-speed coupling excitation model called 7-DOF nonlinear full-vehicle model and differential equation are established by introducing the time delay of incentive input and then by using numerical simulation to analyze chaotic vibration in 7-DOF nonlinear full-vehicle model excited by consecutive speed control humps. The numerical simulation results show that chaotic vibration phenomenon possibly appears as vehicles are driven on consecutive speed control humps. Further studies indicate that the influence of nonlinear running state of vehicle on driving comfort becomes manifest as the state changes from chaotic motions to the periodic motion of the high speed and the periodic motion of the low speed, and this phenomenon can be avoided by changing the parameters of consecutive speed control humps. The results can be applied in design of vehicle and road humps pavement.
\end{abstract}

\section{Introduction}

Road speed control humps are a kind of forced road traffic safety facilities, which play an important role in curbing the occurrence of traffic accidents [1,2]. At present, all various speed control bumps are paved on road; in addition to the common entrance, community and school road install rubber speed control bumps and the accident black spot on highway, such as highway curve, ramp, and tunnel entrances, generally setting up consecutive speed bumps to force the vehicle deceleration. When the car passes the uneven speed control bumps, severe vibration through the body and seat is passed from tire to the driver, which makes the driver feel uncomfortable. Thus, the driver will be forced to reduce speed to achieve the goal of speed limit [3-5].

The automobile suspension system is necessary equipment, which can reduce the vibration generated by the
SCHs, improve the vehicle's driving smoothness and comfort, and reduce the damage of the vehicle and road surface. Because of the nonlinearities of automobile suspension system and wheels, the nonlinear characteristics existing in the automobile suspension system can lead to suspension system producing complicated dynamic behaviors under uneven road surface excitation, such as chaotic vibration and quasiperiodic vibration. When the vibration becomes sever, it will lead the vehicle to jump or overturn $[3,6,7]$. Chaotic vibration and quasiperiodic vibration may cause shock vibration to road surface, thus influencing the lifetime of road surface, safety of driving, and the comfort of driver [8-13].

In recent years, the nonlinear complex dynamic behavior of the automobile suspension system caused by road roughness excitation has caused more and more concerns [2-17]. This study mainly focuses on the single-DOF, two-DOF, and 


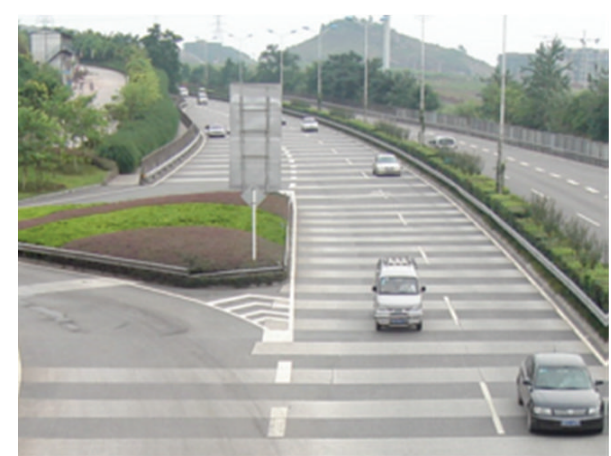

(a) SCHs in highway turn section or ramp

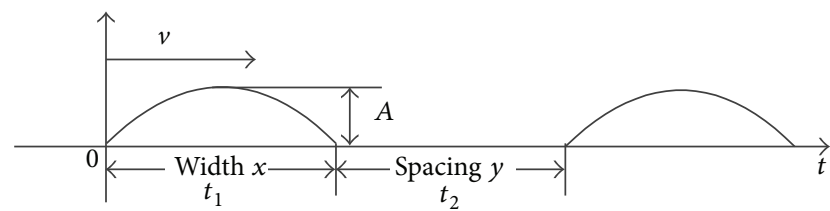

(b) Consecutive SCHs excitation

FIGURE 1: Consecutive SCHs excitation.

four-DOF vehicle models and analyzes the chaotic vibration induced by road excitation, which includes sine wave excitation and multifrequency and multiamplitude or random excitation. The traditional vehicle dynamics models are mainly divided into $1 / 4$ vehicle model and half-vehicle model. 2DOF $1 / 4$ vehicle model is a simple vehicle dynamics model which just studies the vertical direction of movement of the vehicle body, and 4-DOF half-vehicle model is relatively close to the actual model of suspension system; it can be used for studying not only vertical movement and pitch motion of the vehicle body, but also the vertical movement of front and rear wheels, so the traditional vehicle dynamics models have some limitations and cannot reflect the overall dynamic behavior of vehicles [18]. In fact, the dynamic response of various models is different obviously; simple models are difficult to provide enough information to reflect the actual dynamic characteristics of suspension system and vehicle body. 7-DOF vehicle 3D model can completely study the vertical movement, pitch, and roll motion of car body and the vertical movement of the status of four wheels, which has high practical value [19]. In practice, the running state of 2-DOF and 4-DOF nonlinear vehicle model excited by consecutive speed control humps had been investigated in domestically and overseas $[5-7,14-16]$, but the chaotic motions of 7DOF nonlinear vehicle model by considering the effect of the different excitation frequency have not yet been developed. Therefore, the speed range inducing chaotic vibration is not clear, as well as the relationship between the parameters of SCHs and chaotic vibration, and the relationship between the nonlinear running state of vehicle and the driving comfort. Aiming at a deceleration road section on highway, the height of SCHs is fixed; when driving in continuous speed control bumps of different speed, the driving period is different, which means the SCHs can bring different excitation frequency to different speed of car. Therefore, we study the action of nonlinear vibration of the automobile suspension system under the fixed amplitude and different frequency areas of road surfaces, and the relationship between the nonlinear running state of vehicle and the driving comfort. It is of great practical significance for designing vehicle and road pavement.
Aiming at this special road of the SCHs in highway, the SCHs-speed coupling excitation model, 7-DOF nonlinear full-vehicle model, and dynamic equation are firstly presented, then the response of nonlinear chaotic vibration of 7-DOF nonlinear full-vehicle model excited by consecutive SCHs is analyzed by numerical simulation, the range of the chaotic vibration frequency is derived, and the relationship between the nonlinear running state of vehicle and the driver's comfort is obtained from the perspective of vibration strength (VS) and vibration amplitude (VA). The numerical simulation shows that the chaotic vibration will appear when vehicles pass consecutive speed control humps under special speed range. Meanwhile, it is concluded that the low periodic motion zone is the strongest region for affecting the vehicle comfort, adjusting the parameters of SCHs to avoid this phenomenon.

\section{The Vehicle Suspension System and the Road Excitation Model}

2.1. The SCHs-Speed Coupling Excitation Model. The SCHs on highway are mainly paved by the periodic or aperiodic form on tunnel portal, turn section, ramp, and so forth. The paper focuses on highway ramp, curve continuous deceleration zones, and survey and found that the SCHs have approximately the same width and spacing, and the form of the SCHs is similar to the shape of half-sine wave. Therefore, the paper uses the half-sine wave to simulate highway SCHs; the physical objects and static model of highway SCHs are shown in Figure 1.

In Figure 1(b), $A$ is the height of the speed control bump, $x$ is width of speed control bump, and $y$ is the distance between two adjacent speed-control bumps. In Figure 1, vehicle goes through a series of consecutive SCHs with speed $v, t_{1}$ is the time for a vehicle to pass a hump, and $t_{2}$ is time for the vehicle through two adjacent speed control humps, so $t_{1}=x / v, t_{2}=$ $y / v$; that is to say, the period $T=t_{1}+t_{2}$; thus the relationship between excitation frequency and speed can be given as $f=$ $v /(x+y)$.

At present, the parameter of SCHs is fixed at no unified standard; the range of $A$ is usually $0.003 \mathrm{~m} \sim 0.020 \mathrm{~m}$; 


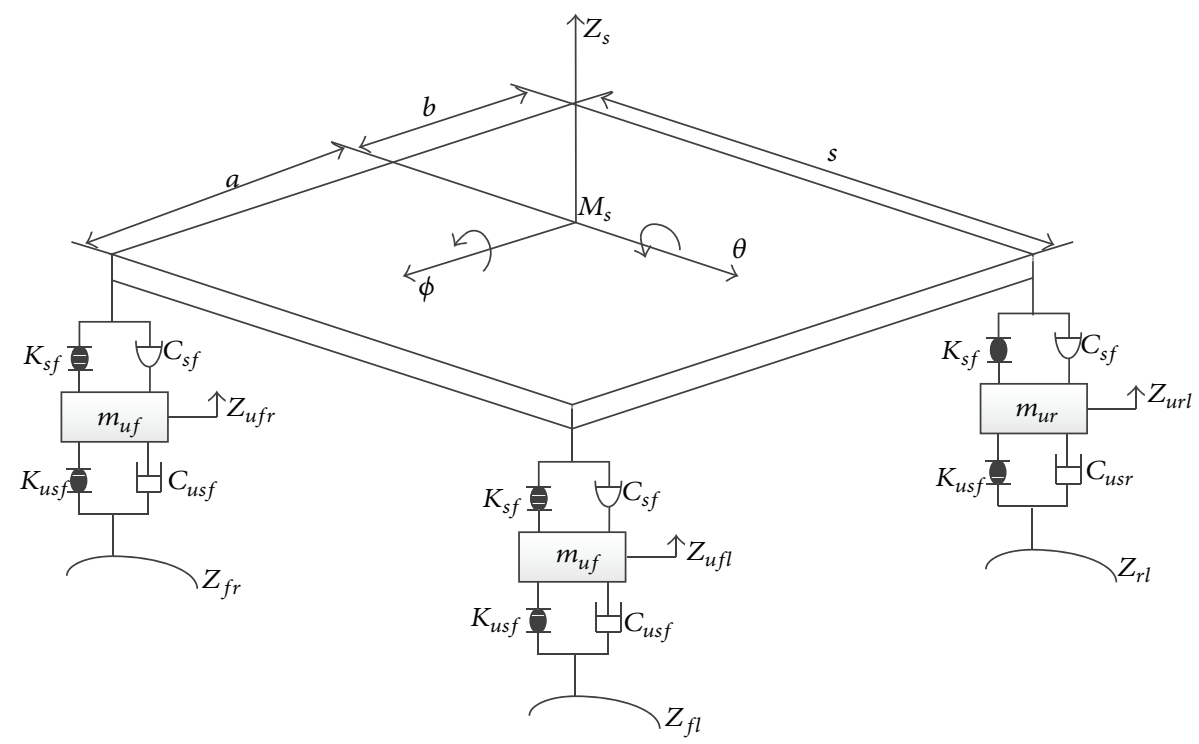

FIGURE 2: Nonlinear seven-DOF full-vehicle model.

$A=0.02 \mathrm{~m}$ is fixed in this paper. Because the road surface roughness is far less than the height of the $\mathrm{SCH}$, we can ignore road roughness of SCHs section, and the waveform of consecutive SCHs in highway is similar to a half-sine wave. According to the field observation measurement, width is equal to space incurve road and ramp; namely, $x=y$. Therefore, the dynamic excitation of consecutive SCHs to vehicles' four-wheel motivation can be defined, respectively:

$$
\begin{gathered}
Z_{f r}(t)= \begin{cases}A \sin \left(\frac{2 \pi v}{x} t\right), & \sin \left(\frac{2 \pi v}{x} t\right)>0, \\
0, & \sin \left(\frac{2 \pi v}{x} t\right) \leq 0,\end{cases} \\
Z_{f l}(t)= \begin{cases}A \sin \left(\frac{2 \pi v}{x} t+\beta\right), & \sin \left(\frac{2 \pi v}{x} t\right)>0, \\
0, & \sin \left(\frac{2 \pi v}{x} t\right) \leq 0,\end{cases} \\
Z_{r r}(t)= \begin{cases}A \sin \left(\frac{2 \pi v}{x} t+\alpha\right), & \sin \left(\frac{2 \pi v}{x} t\right)>0, \\
0, & \sin \left(\frac{2 \pi v}{x} t\right) \leq 0,\end{cases} \\
Z_{r l}(t)= \begin{cases}A \sin \left(\frac{2 \pi v}{x} t+\alpha+\beta\right), & \sin \left(\frac{2 \pi v}{x} t\right)>0, \\
0, & \sin \left(\frac{2 \pi v}{x} t\right) \leq 0,\end{cases}
\end{gathered}
$$

where $A$ and $f$ denote amplitude and frequency of the sine excitation in consecutive SCHs section, respectively; $\beta$ denotes incentives time delay of excitation to the two front or two rear wheels of vehicle; $\alpha$ denotes incentives time delay of excitation to the vehicle's left and right front wheels.

2.2. Seven-DOF Full-Vehicle Model. A nonlinear vehicle dynamics simulation model which can reflect the real running state of vehicle and full-DOF is established. In Figure 2
[19], the vehicle body can consist of a three-DOF $(\phi, \theta$, and $Z_{s}$ in Figure 1) and cuboid $m_{s}$. Ignoring the vehicle's yaw, rotational degree of freedom, and steering angle of four wheels, vehicle body has three other characteristics that are pitch roll, vertical jump, and the four wheels which can vertically leap.

Symbols are defined in Figure 2 as follows: $m_{s}$ : sprung mass; $I_{\phi}$ : roll axis moment of inertia; $I_{\theta}$ : pitch axis moment of inertia; $m_{u f}, m_{u r}$ : front/rear unsprung mass; $k_{s f}, k_{s r}$ : front/rear suspension spring stiffness; $c_{s u f}, c_{s d f}$ : damping coefficient of front suspension; $c_{s u r}, c_{c d r}$ : damping coefficient of rear suspension; $k_{u s f}, k_{u s r}$ : tire spring stiffness; $c_{u s f}, c_{u s r}$ : damping coefficient of tire; $a$ : length between the front of vehicle and the center of gravity of sprung mass; $b$ : length between the rear of vehicle and the center of gravity of sprung mass; $s$ : width of sprung mass.

Suspension spring damping unit has nonlinear characteristics, ignoring the influence of tire damping at the same time, just considering the nonlinear mechanical properties of elastic force. It is assumed that the modeled nonlinear spring of suspension has the following characteristics $[18,19]$ :

$$
F_{s i j}=k_{s i} \operatorname{sgn}\left(\Delta_{s i j}\right)\left|\Delta_{s i j}\right|^{n_{s i}} \quad(i=f, r ; j=l, r) .
$$

The nonlinear damping forces of suspensions are given by

$$
\begin{gathered}
F_{c i j}=c_{s i} \dot{\Delta}_{u i j} \quad(i=f, r ; j=\ell, r), \\
c_{s i}=\left\{\begin{array}{ll}
\zeta_{i j}, & \dot{\Delta}_{i j} \geq 0, \\
\xi_{i j}, & \dot{\Delta}_{i j}<0,
\end{array} \quad(i=f, r ; j=\ell, r) .\right.
\end{gathered}
$$

The nonlinear characteristic of vehicle's tire is

$$
F_{u s i j}=k_{u s i} \operatorname{sgn}\left(\Delta_{u s i j}\right)\left|\Delta_{u s i j}\right|^{n_{u s i}} \quad(i=f, r ; j=\ell, r),
$$

where $k_{s i}$ is the equivalent stiffness of spring force, $\Delta_{s i j}$ is the deformation, and $n_{s i}$ is the nonlinear coefficient of the tire spring. 
The damping of the tires is assumed to be viscous; thus the damping force is calculated as

$$
F_{u c i j}=c_{u s i} \dot{\Delta}_{u s i j}
$$

where $c_{u s i}$ is the viscous damping coefficient and $\dot{\Delta}_{u s i j}$ is the relative velocity of damper.

Based on the above analysis, the established nonlinear suspension model, and the road excitation condition, in the initial coordinate system o- $x y z$, the nonlinear dynamic equation of 7-DOF full-vehicle by Lagrange method can be established as follows:

$$
\begin{gathered}
m_{s} \ddot{z}_{s}=-F_{s f l}-F_{c f l}-F_{s f r}-F_{c f r} \\
-F_{s r l}-F_{c r l}-F_{s r r}-F_{c r r}-m_{s} g, \\
I_{\varphi} \ddot{\varphi}=\left(-F_{s f l}-F_{c f l}+F_{s f r}+F_{c f r}\right. \\
\left.-F_{s r l}-F_{c r l}+F_{s r r}+F_{c r r}\right) \frac{s}{2} \cos \varphi, \\
I_{\theta} \ddot{\theta}=\left(F_{s f l}+F_{c f l}+F_{s f r}+F_{c f r}\right) a \cos \theta \\
-\left(F_{s r l}+F_{c r l}+F_{s r r}+F_{c r r}\right) b \cos \theta, \\
m_{u f} \ddot{z}_{u f l}=F_{s f l}+F_{c f l}-F_{u s f l}-F_{u c f l}-m_{u f} g, \\
m_{u f} \ddot{z}_{u f r}=F_{s f r}+F_{c f r}-F_{u s f r}-F_{u c f r}-m_{u f} g, \\
m_{u r} \ddot{z}_{u r l}=F_{s r l}+F_{c r l}-F_{u s r l}-F_{u c r l}-m_{u r} g, \\
m_{u r} \ddot{z}_{u r r}=F_{s r r}+F_{c r r}-F_{u s r r}-F_{u c r r}-m_{u r} g .
\end{gathered}
$$

The forces related to the sprung mass in (6) can be calculated as

$$
\begin{gathered}
F_{s f l}=100^{\left(n_{s f}-1\right)} k_{s f} \operatorname{sgn}\left(\Delta_{u f l}-\Delta_{s f}\right)\left|\Delta_{u f l}-\Delta_{s f}\right|^{n_{s f}}, \\
F_{s f r}=100^{\left(n_{s f}-1\right)} k_{s f} \operatorname{sgn}\left(\Delta_{u f r}-\Delta_{s f}\right)\left|\Delta_{u f r}-\Delta_{s f}\right|^{n_{s f}}, \\
F_{c f l}=c_{s f} \dot{\Delta}_{u f l}, \\
F_{c f r}=c_{s f} \dot{\Delta}_{u f r}, \\
F_{s r l}=100\left(n_{s r}-1\right) k_{s r} \operatorname{sgn}\left(\Delta_{u r l}-\Delta_{s r}\right)\left|\Delta_{u r l}-\Delta_{s r}\right|^{n_{s r}}, \\
F_{s r r}=100\left(n_{s r}-1\right) k_{s r} \operatorname{sgn}\left(\Delta_{u r r}-\Delta_{s r}\right)\left|\Delta_{u r r}-\Delta_{s r}\right|^{n_{s r}}, \\
F_{c r l}=c_{s r} \dot{\Delta}_{u r l}, \\
F_{c r r}=c_{s r} \dot{\Delta}_{u r r},
\end{gathered}
$$

where

$$
\begin{gathered}
\Delta_{u f l}=\frac{s}{2} \sin \varphi-a \sin \theta+z_{s}-z_{u f l}, \\
\Delta_{u f r}=-\frac{s}{2} \sin \varphi-a \sin \theta+z_{s}-z_{u f r}, \\
\Delta_{u r l}=\frac{s}{2} \sin \varphi+b \sin \theta+z_{s}-z_{u r l}, \\
\Delta_{u r r}=-\frac{s}{2} \sin \varphi+b \sin \theta+z_{s}-z_{u r r}
\end{gathered}
$$

$$
\begin{aligned}
\Delta_{s r} & =\left[\frac{a}{100^{\left(n_{s r}-1\right)} k_{s r}}\left(\frac{m_{s} g}{2(a+b)}\right)\right]^{1 / n_{s r}}, \\
\Delta_{s f} & =\left[\frac{b}{100^{\left(n_{s f}-1\right)} k_{s f}}\left(\frac{m_{s} g}{2(a+b)}\right)\right]^{1 / n_{s f}} .
\end{aligned}
$$

The forces related to the unsprung mass can be expressed as follows:

$$
\begin{gathered}
F_{u s f l}=100^{\left(n_{f}-1\right)} k_{u s f} \operatorname{sgn}\left(\Delta_{u s f l}-\Delta_{s u f}\right)\left|\Delta_{u s f l}-\Delta_{s u f}\right|^{n_{u s f}}, \\
F_{u s f r}=100^{\left(n_{f}-1\right)} k_{u s f} \operatorname{sgn}\left(\Delta_{u s f r}-\Delta_{s u f}\right)\left|\Delta_{u s f r}-\Delta_{s u f}\right|^{n_{u s f}}, \\
F_{u c f l}=c_{u s f} \dot{\Delta}_{u s f l}, \\
F_{u c f r}=c_{u s f} \dot{\Delta}_{u s f r}, \\
F_{u s r l}=100^{\left(n_{r}-1\right)} k_{u s r} \operatorname{sgn}\left(\Delta_{u s r l}-\Delta_{s u r}\right)\left|\Delta_{u s r l}-\Delta_{s u r}\right|^{n_{u s r}}, \\
F_{u s r r}=100^{\left(n_{r}-1\right)} k_{u s r} \operatorname{sgn}\left(\Delta_{u s r r}-\Delta_{s u r}\right)\left|\Delta_{u s r r}-\Delta_{s u r}\right|^{n_{u s r}}, \\
F_{u c r l}=c_{u s r} \dot{\Delta}_{u s r l}, \\
F_{u c r r}=c_{u s r} \dot{\Delta}_{u s r r},
\end{gathered}
$$

where

$$
\begin{gathered}
\Delta_{u s f l}=z_{u f l}-\frac{(A \sin (\omega t)+|A \sin (\omega t)|)}{2}, \\
\Delta_{u s f r}=z_{u f r}-\frac{(A \sin (\omega t)+|A \sin (\omega t)|)}{2}, \\
\Delta_{u s r l}=z_{u r l}-\frac{(A \sin (\omega t+\alpha)+|A \sin (\omega t+\alpha)|)}{2}, \\
\Delta_{u s r r}=z_{u r r}-\frac{(A \sin (\omega t+\alpha)+|A \sin (\omega t+\alpha)|)}{2}, \\
\Delta_{s u r}=\left[\frac{g}{100^{\left(n_{r}-1\right)} k_{s u r}}\left(\frac{m_{s} a}{2(a+b)}+m_{u r}\right)\right]^{1 / n_{u s r}}, \\
\Delta_{s u f}=\left[\frac{g}{100^{\left(n_{f}-1\right)} k_{s u f}}\left(\frac{m_{s} b}{2(a+b)}+m_{u f}\right)\right]^{1 / n_{u s f}} .
\end{gathered}
$$

\section{Numerical Simulation}

Based on the above established nonlinear dynamics model and system dynamic equations (6), the dynamic response of the system was studied numerically using the forth order Runge-Kutta algorithm provided by MATLAB. Since numerical integration could give spurious results regarding the existence of chaos due to relative big time steps, the step length was verified to ensure that no such solutions were generated as a result of time discretization.

The parameters of the full-vehicle model which are used in the numerical study are shown in Table 1 [19]. 
TABLE 1: Parameters for numerical simulation.

\begin{tabular}{lccccc}
\hline Parameter & Value & Parameter & Value & Parameter & Value \\
\hline$m_{s}$ & $1500 \mathrm{~kg}$ & $n_{s f}, n_{s r}$ & 1.5 & $n_{u s f}, n_{u s r}$ & 1.25 \\
$I_{\phi}$ & $460 \mathrm{~kg} \mathrm{~m}$ & $c_{s u f}$ & $1000 \mathrm{~N} / \mathrm{m} / \mathrm{s}$ & $a$ & $1.4 \mathrm{~m}$ \\
$I_{\theta}$ & $2160 \mathrm{~kg} \mathrm{~m}$ & $c_{s d f}$ & $720 \mathrm{~N} / \mathrm{m} / \mathrm{s}$ & $b$ & $1.7 \mathrm{~m}$ \\
$m_{u f}$ & $59 \mathrm{~kg}$ & $c_{s u r}$ & $1000 \mathrm{~N} / \mathrm{m} / \mathrm{s}$ & $s$ & $3 \mathrm{~m}$ \\
$m_{u r}$ & $59 \mathrm{~kg}$ & $c_{c d r}$ & $720 \mathrm{~N} / \mathrm{m} / \mathrm{s}$ & $\alpha$ & $58^{\circ}$ \\
$k_{s f}$ & $35,000 \mathrm{~N} / \mathrm{m}$ & $k_{u s f}, k_{u s r}$ & $190,000 \mathrm{~N} / \mathrm{m}$ & $\beta$ & $9^{\circ}$ \\
$k_{s r}$ & $38,000 \mathrm{~N} / \mathrm{m}$ & $c_{u s f}, c_{u s r}$ & $10 \mathrm{~N} / \mathrm{m} / \mathrm{s}$ & & \\
\hline
\end{tabular}

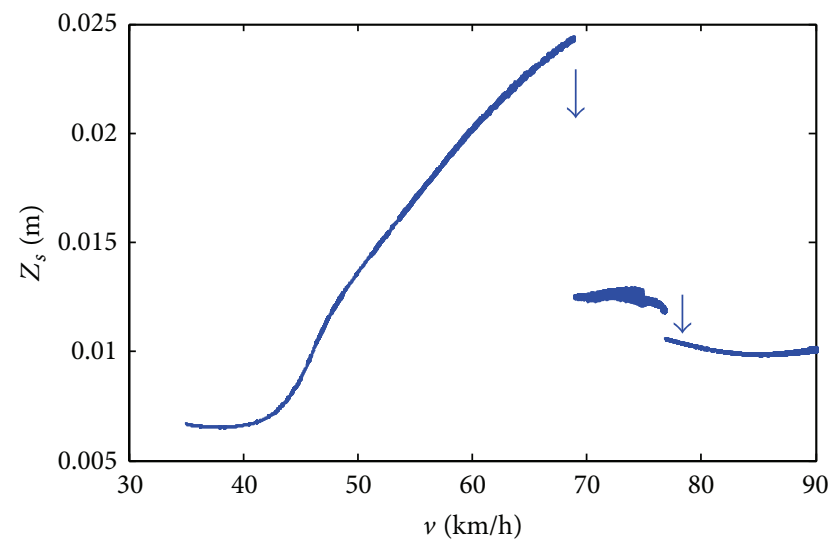

Figure 3: The speed of vertical displacement $Z_{s}$ bifurcation diagram.

3.1. The Nonlinear Dynamics Analysis of System. In general, we use bifurcation diagram of the amplitude of vibration system relative to the excitation frequency change to analyze the dynamic response of system; the maximum value of displacement amplitude's absolute value is defined as system amplitude. For the car driven on the continuous $\mathrm{SCH}$, speed is more obvious, so the paper uses response of the figure (bifurcation diagram) of the variation amplitude relative to the speed to analyze system dynamic response. In the dynamic system, bifurcation diagram is commonly used to check the response diagram under certain parameter change of model. In this paper, road surface excitation frequency (the speed of the vehicle driven on the SCHs) can be taken as a parameter in bifurcation diagram, which is obtained by plotting Poincaré points, as shown in Figures 3-6.

In the simulation, the amplitude $A$ of SCHs is $0.02 \mathrm{~m}$, the speed range is $35.00 \mathrm{~km} / \mathrm{h}<V<90 \mathrm{~km} / \mathrm{h}$, and speed change step is $0.001 \mathrm{~km} / \mathrm{h}$. Figures 3-6 show the frequency response, which are vertical displacement of the sprung mass, rotating angle of sprung mass around its center, pitch angle of the vehicle, and four wheels' vertical displacement diagram (because the four wheels are symmetrical, we just need to analyze the front-left wheel movement condition), respectively. Simulation time in system is $20 \mathrm{~s}$, the integral time of solving the dynamic differential equations is 1000 cycles, the last 200 Poincaré points are plotted by taking incentive period points in each to determine the bifurcation parameter, and the original 1600 incentive Poincaré points

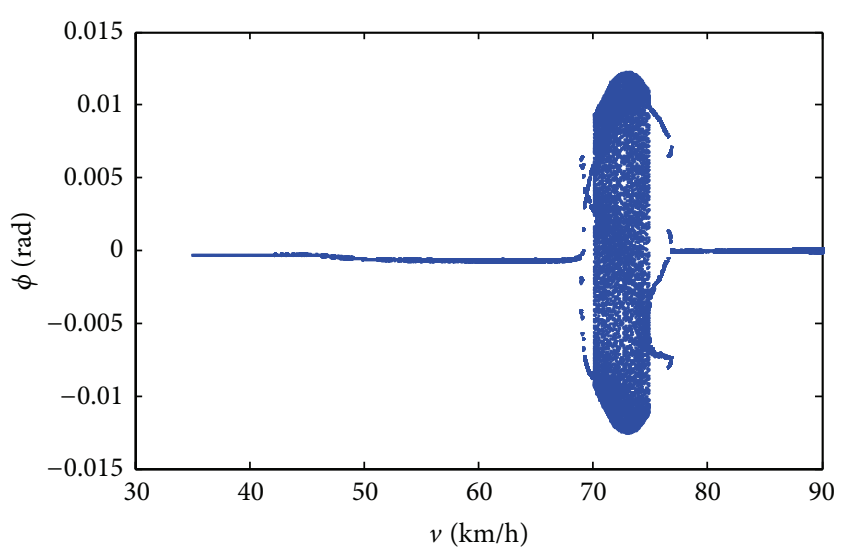

FIGURE 4: Speed bifurcation diagram of $\phi$.

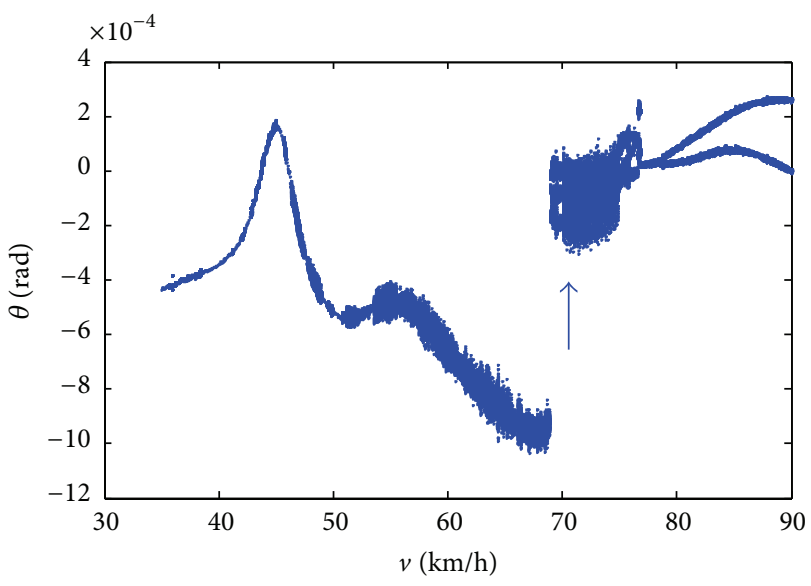

FIGURE 5: Speed bifurcation diagram of $\theta$.

data are not shown in the diagram, which can guarantee the used data in vibration stability state, thus avoiding the influence of transient response.

Figure 3 shows bifurcation diagram of the car body's vertical displacement $Z_{s}$ continuous changes with vehicle speed change. When the vehicle speed increases gradually, there was a downward jump as $v=68.85 \mathrm{~km} / \mathrm{h}$ and $v=76.80 \mathrm{~km} / \mathrm{h}$. When between $v=69.05 \mathrm{~km} / \mathrm{h}$ and $v=76.80 \mathrm{~km} / \mathrm{h}$, motion state of system changed from the oscillations to beats; the area is the unstable regions of the system; this phenomenon in nonlinear dynamic response analysis of 4-DOF nonlinear half-vehicle model and 2-DOF quarter car model cannot be obtained.

Figure 4 represents dynamic responses diagram of roll movement of vehicle. According to the characteristics in Figure 4, it can be divided into three regions: A (35.00 $68.85 \mathrm{~km} / \mathrm{h}), B(68.85 \sim 77.05 \mathrm{~km} / \mathrm{h})$, and C $(77.05 \sim 95 \mathrm{~km} / \mathrm{h})$. It is clearly found that the bifurcation diagram of vehicle roll movement and the vertical displacement of car body are obviously different, without obvious upward or downward leap. In region $B$, vehicles do beat movement. The existence of the unstable region means that the system is possibly producing chaotic motion phenomenon. 
TABLE 2: Amplitude under different nonlinear characteristics.

\begin{tabular}{lcc}
\hline Nonlinear characteristics area & Amplitude range of $z_{s}(\mathrm{~m})$ & Vibration intensity range of $z_{s}(\mathrm{~g})$ \\
\hline Periodic A $(35.00 \sim 68.85 \mathrm{~km} / \mathrm{h})$ & $0.002011 \sim 0.003463$ & $0.004099 \sim 0.01934$ \\
Chaotic B $(68.85 \sim 76.80 \mathrm{~km} / \mathrm{h})$ & $0.0002934 \sim 0.0007994$ & $0.000000 \sim 0.001935$ \\
Periodic C $(76.80 \sim 90.00 \mathrm{~km} / \mathrm{h})$ & $0.0000000 \sim 0.0007626$ & $0.00000 \sim 0.004356$ \\
\hline
\end{tabular}

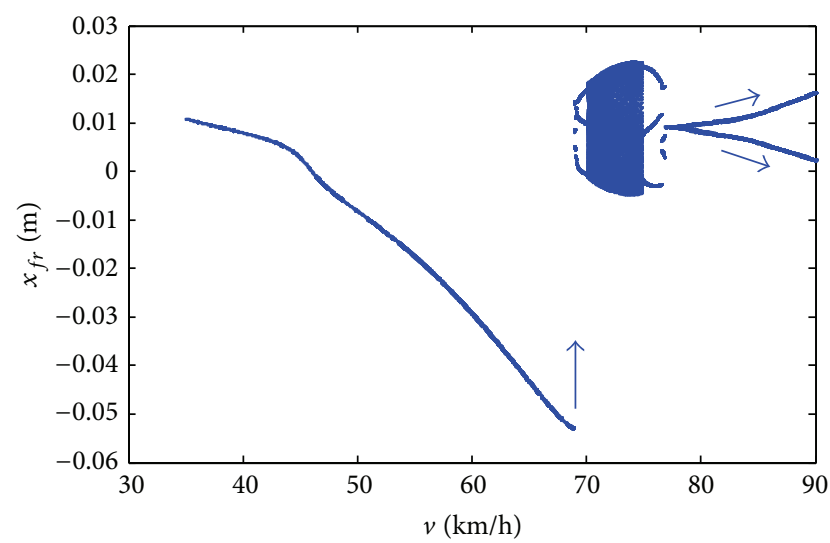

FIGURE 6: The bifurcation diagram of the speed of front-rear wheels.

Jump up and bifurcation (fractal) phenomenon can be obtained in the dynamic response diagram of the pitch motion of the car. In Figure 5, we can obtain that when $v=68.85 \mathrm{~km} / \mathrm{h}$, an upward jump appears and when $v=$ $78.75 \mathrm{~km} / \mathrm{h}$, bifurcation (fractal) phenomenon appears. The unstable region exists in $50.85<v<68.85 \mathrm{~km} / \mathrm{h}$ and $69.05 \mathrm{~km} / \mathrm{h}<v<76.95 \mathrm{~km} / \mathrm{h}$; and $50.85 \mathrm{~km} / \mathrm{h}<v<$ $68.85 \mathrm{~km} / \mathrm{h}$ is the new additional region.

Since front- and rear-wheels of the car are symmetrical and the left and right wheels are symmetrical, the sprung mass of four wheels has the same movement characteristics. Therefore, we only plot the bifurcation diagram of vehicle front-left wheel, as shown in Figure 6. From Figure 6, when $v=68.85 \mathrm{~km} / \mathrm{h}$, there exists a big jump; when $v=$ $77.95 \mathrm{~km} / \mathrm{h}$, the system has a bifurcation (fractal) phenomenon. The instability area of the system did not increase. From the bifurcation diagram, we can see that the wheel movement has similar dynamic response to the pitch angle of vehicle body in Figure 5 .

Through analyzing of the bifurcation diagram of 7-DOF nonlinear vehicle, we can find that movement characteristics of the system are similar in the same area. At the same time, chaos movement might appear in the unstable region and may appear in the jump area. The unstable areas are analyzed by using the Poincaré, phase diagram, and PSP to further study system and whether there exists chaos motion in a given area, as shown in Figure 7.

When $v=72.65 \mathrm{~km} / \mathrm{h}$, system movement mode is shown in Figure 4. Phase diagram can consist of multiple closed curves, and these are filled in the whole phase space, Poincaré section has an unlimited number of points, PSP value in each turbulence period is approximately random, and cloud structure of its point has no rules, which means that the system does chaotic motions.

\section{The Influence of Analysis of Nonlinear Vibration on Passenger Comfort}

Road control bumps are one of the main factors to influence the vehicle driving comfort. When car is driven, some factors such as road roughness make car vibration. The vibration of the vehicle transmits from suspension, bottom deck, and the seat to driver, which can cause human body vibration and lead to the passenger's comfort becoming lower. The researches show that the human response to vibration related to vibration direction, frequency, intensity, and exposure time $[4,6,7]$. In general, internal vibration of the vehicle suspension system can be denoted by VA and intensity VS, and the vibration intensity and vibration amplitude are the main factors to influence the comfort, so the paper applies the nonlinear vibration amplitude and vibration intensity to analyze the effect of nonlinear characteristic on the comfort of vehicle suspension systems.

We use PSP value of system state parameters under the influence of different nonlinear characteristics to reflect the vibration characteristics of the system. As shown in Figures 8 and 9 , the characteristics of vertical vibration of vehicle body in speed range of $35.00 \sim 90.00 \mathrm{~km} / \mathrm{h}$ are shown in PSP-speed bifurcation diagram and VS-speed bifurcation diagram.

Figures 8 and 9 are the speed of the bifurcation diagram; in the speed area of the different nonlinear characteristics, the amplitude PSP value of the vehicle body vertical movement under different nonlinear characteristics and vibration intensity range is shown in Table 2 .

Figure 8 illustrates that when the vehicle speed ranges in region $\mathrm{A}$, the vibration change amplitude of vehicle body vertical direction is maximum, while amplitudes of other areas are lower. It can be found in Figure 9 that the presented characteristics of VS-speed bifurcation diagram and corresponding PSP-speed bifurcation diagram of system vibration parameters have great similarities, also period state can affect vehicle vibration intensity obviously, and influence of chaos on vehicle vibration is relatively weak. For the influence of analysis of different nonlinear dynamic characteristics on internal vibration of suspension system, we can deduce that, in produced periodic motion area of the system, namely, speed in the periodic A $(35.00$ $68.85 \mathrm{~km} / \mathrm{h}$ ), nonlinear characteristic of system can lead to the vehicle vibration intensifying, which can seriously influence the driving comfort and safety. When speeding in other areas, the vehicle vibration intensity and amplitude were 

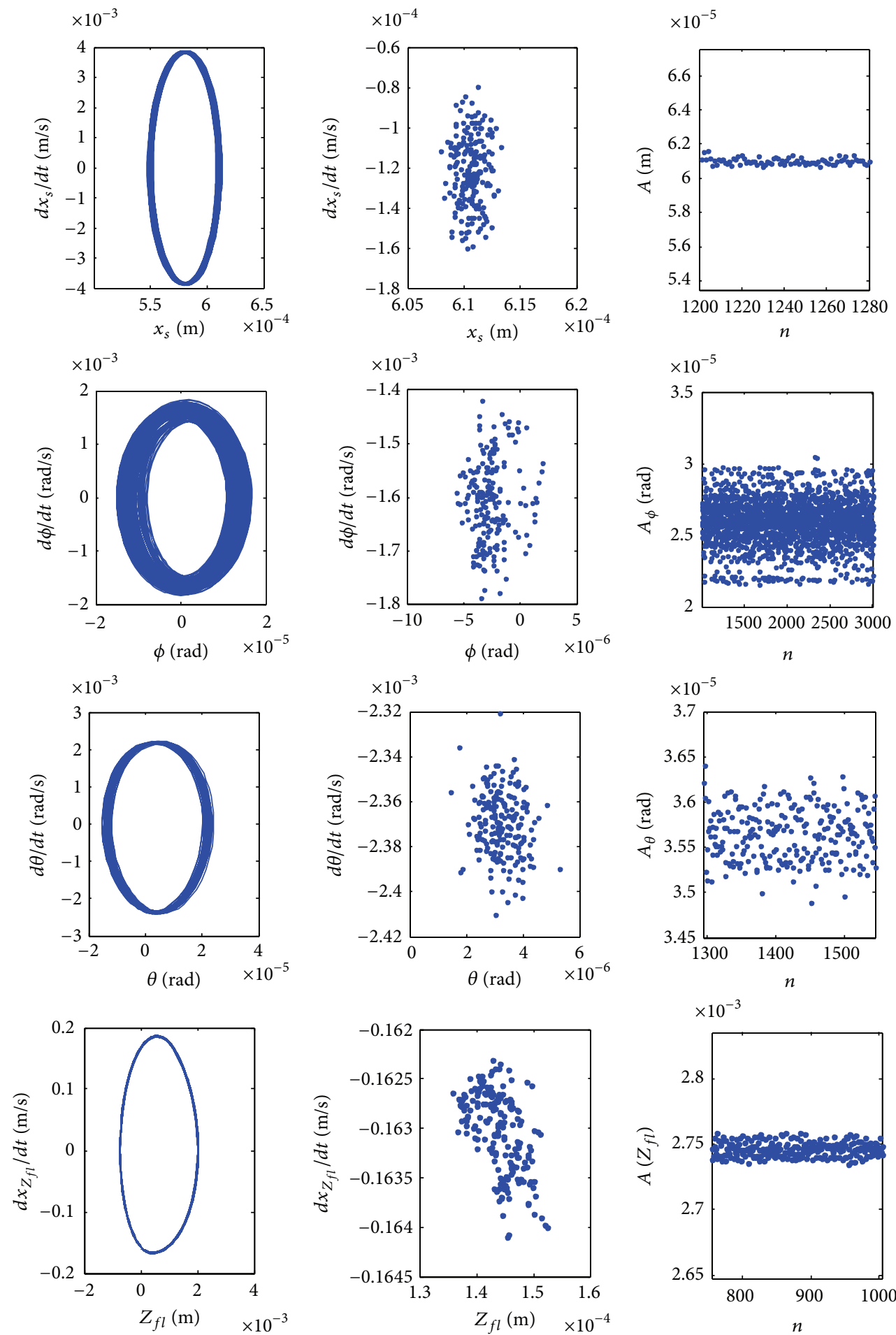

(a)

(b)

(c)

Figure 7: Chaotic motion $(v=72.65 \mathrm{~km} / \mathrm{h})$. 


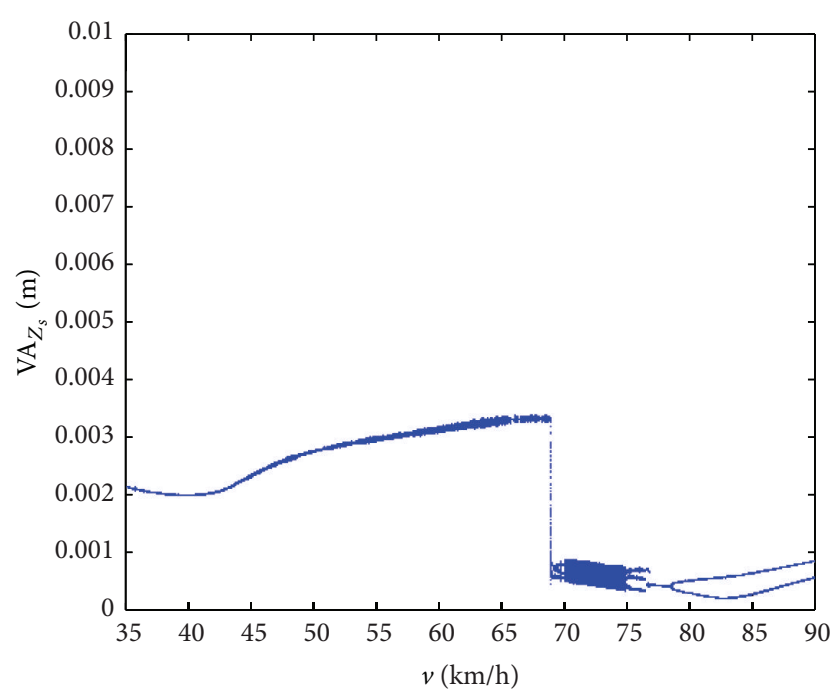

FiguRE 8: PSP-speed bifurcation diagram of $z_{s}$.

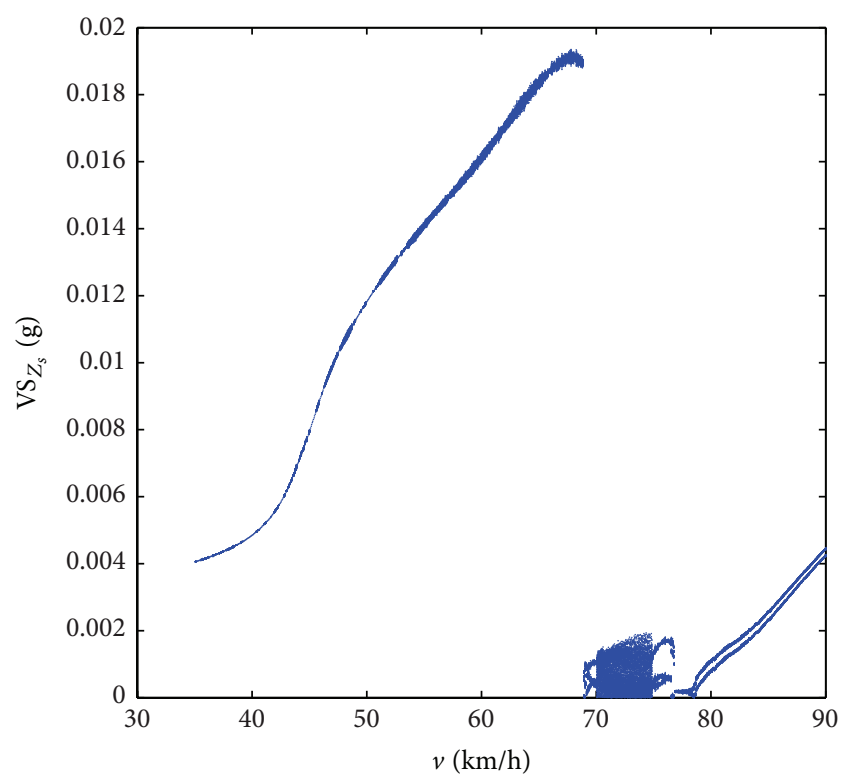

FIGURE 9: VS-speed bifurcation diagram of $z_{s}$.

decreased significantly. This special nonlinear phenomenon can provide important reference significance to design of bumps pavement and road speed limit parameter.

\section{Conclusion}

In this paper, a half-sine wave excitation is applied to simulate highway SCHs, and 7-DOF nonlinear vehicle model is established. Under the condition of four wheels with time delay, chaotic vibration of 7-DOF nonlinear vehicle model and the relationship between vibration and comfort are researched, and the complex dynamic behavior of the system is obtained by numerical simulation. Applying bifurcation diagram, we found that when the speed of the vehicle reaches a certain range, the instability regions of system appear, while the phenomenon in the study of 2-DOF and 4-DOF vehicle model could not be obtained. The chaotic phenomena occur in 4 -DOF model when vehicle speed is between $59.00 \mathrm{~km} / \mathrm{h} \sim$ $70.00 \mathrm{~km} / \mathrm{h}$ occur in 4 -DOF model as the range of the vehicle speed under $68.85 \sim 76.80 \mathrm{~km} / \mathrm{h}$, which initial velocity of the vehicle larger than that of 4 -DOF in chaotic phenomena. Compared with the 4-DOF model, the results of 7-DOF model are closer to the real situation, which has greater practical value. The comparison between two models can be seen in the paper conclusions. The influence of the nonlinear vibration state of vertical direction on comfort is studied; the results show that the vehicle's vibration intensity is the largest in low periodic motion state, and the influence of the nonlinear vibration on driving comfort is the biggest.

Although the vehicle dynamic model is just a simplified model and we selected control parameters with certain difference from the actual data, our results are still effective in the dynamics of vehicle dynamic design; meanwhile, the results may still provide a certain reference for the vehicle driven on the SCHs with appropriate speed. In the next step, we will further study the influence of various parameters of consecutive SCHs on the system dynamics behavior, and chaos has been tested in the actual system.

\section{Conflict of Interests}

The authors declare that there is no conflict of interests regarding the publication of this article.

\section{Acknowledgments}

The authors would like to thank anonymous referees for their helpful comments and suggestions and the Fundamental Research Funds for the Central Universities (CDJXS12170002) and the Fundamental Research Funds for the Board of Education in Chongqing Municipality (KJ112002) for support.

\section{References}

[1] F. Liu, S. Liang, Q. Zhu, and Q. Xiong, "Effects of the consecutive speed humps on chaotic vibration of a nonlinear vehicle model," ICIC Express Letters, vol. 4, no. 5, pp. 1657-1664, 2010.

[2] B. Antić, D. Pešić, M. Vujanić, and K. Lipovac, "The influence of speed bumps heights to the decrease of the vehicle speedBelgrade experience," Safety Science, vol. 57, pp. 303-312, 2013.

[3] S. Liang, C. Li, Q. Zhu, and Q. Xiong, "The influence of parameters of consecutive speed control humps on the chaotic vibration of a 2 -DOF nonlinear vehicle model," Journal of Vibroengineering, vol. 13, no. 3, pp. 406-413, 2011.

[4] X. Wang and Y. He, "Projective synchronization of fractional order chaotic system based on linear separation," Physics Letters A, vol. 372, no. 4, pp. 435-441, 2008.

[5] X. Wang, X. Zhang, and C. Ma, "Modified projective synchronization of fractional-order chaotic systems via active sliding mode control," Nonlinear Dynamics, vol. 69, no. 1-2, pp. 511-517, 2012.

[6] S. Liang, Y. Sun, Q. Zhu, and Z. Yang, "Ride comfort analysis of a nonlinear vehicle excited by the consecutive speed-control 
humps," Journal of Vibroengineering, vol. 15, no. 4, pp. 16681676, 2013.

[7] Z. Yang, S. Liang, Y. Sun, and Q. Zhu, "Chaos of a Nonlinear half-vehicle suspension system excited by the consecutive speed-control humps," ICIC Express Letters, vol. 7, no. 11, pp. 3163-3168, 2013.

[8] A. Sezgin and Y. Z. Arslan, "Analysis of the vertical vibration effects on ride comfort of vehicle driver," Journal of Vibroengineering, vol. 14, no. 2, pp. 559-571, 2012.

[9] L. Zuo and P.-S. Zhang, "Energy harvesting, ride comfort, and road handling of regenerative vehicle suspensions," Journal of Vibration and Acoustics, Transactions of the ASME, vol. 135, no. 1, Article ID 011002, 2013.

[10] X. Wang and Y. Wang, "Adaptive control for synchronization of a four-dimensional chaotic system via a single variable," Nonlinear Dynamics, vol. 65, no. 3, pp. 311-316, 2011.

[11] Y.-J. Liu and Y.-Q. Zheng, "Adaptive robust fuzzy control for a class of uncertain chaotic systems," Nonlinear Dynamics, vol. 57, no. 3, pp. 431-439, 2009.

[12] D. J. Li, "Adaptive output feedback control of uncertain nonlinear chaotic systems based on dynamic surface control technique," Nonlinear Dynamics, vol. 68, no. 1-2, pp. 235-243, 2012.

[13] Y.-J. Liu, C. L. P. Chen, G.-X. Wen, and S. Tong, "Adaptive neural output feedback tracking control for a class of uncertain discrete-time nonlinear systems," IEEE Transactions on Neural Networks, vol. 22, no. 7, pp. 1162-1167, 2011.

[14] Q. Zhu and M. Ishitobi, "Chaos and bifurcations in a nonlinear vehicle model," Journal of Sound and Vibration, vol. 275, no. 3-5, pp. 1136-1146, 2004.

[15] G. Litak, M. Borowiec, M. I. Friswell, and W. Przystupa, "Chaotic response of a quarter car model forced by a road profile with a stochastic component," Chaos, Solitons \& Fractals, vol. 39, no. 5, pp. 2448-2456, 2009.

[16] S. Li, S. Yang, and W. Guo, "Investigation on chaotic motion in hysteretic non-linear suspension system with multi-frequency excitations," Mechanics Research Communications, vol. 31, no. 2, pp. 229-236, 2004.

[17] J. Marzbanrad, P. Poozesh, and M. Damroodi, "Improving vehicle ride comfort using an active and semi-active controller in a half-car model," Journal of Vibration and Control, vol. 19, no. 9, pp. 1357-1377, 2013.

[18] W. Wang, G. Li, and Y. Song, "Nonlinear dynamic analysis of the whole vehicle on bumpy road," Transactions of Tianjin University, vol. 16, no. 1, pp. 50-55, 2010.

[19] Q. Zhu and M. Ishitobi, "Chaotic vibration of a nonlinear fullvehicle model," International Journal of Solids and Structures, vol. 43, no. 3-4, pp. 747-759, 2006. 


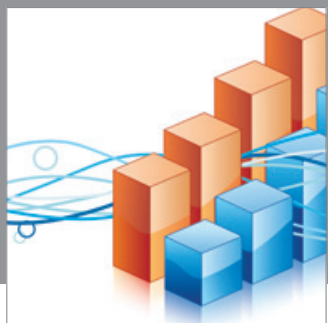

Advances in

Operations Research

mansans

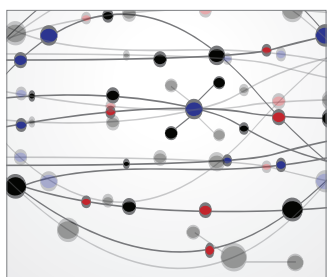

The Scientific World Journal
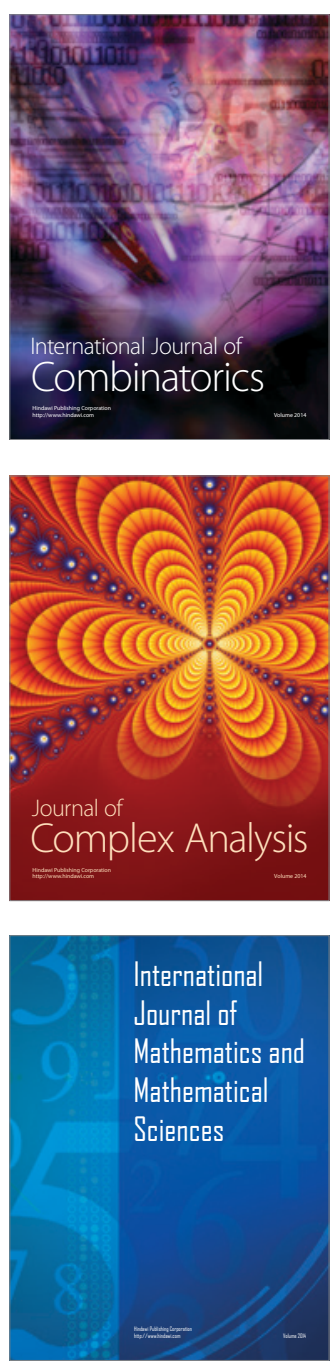
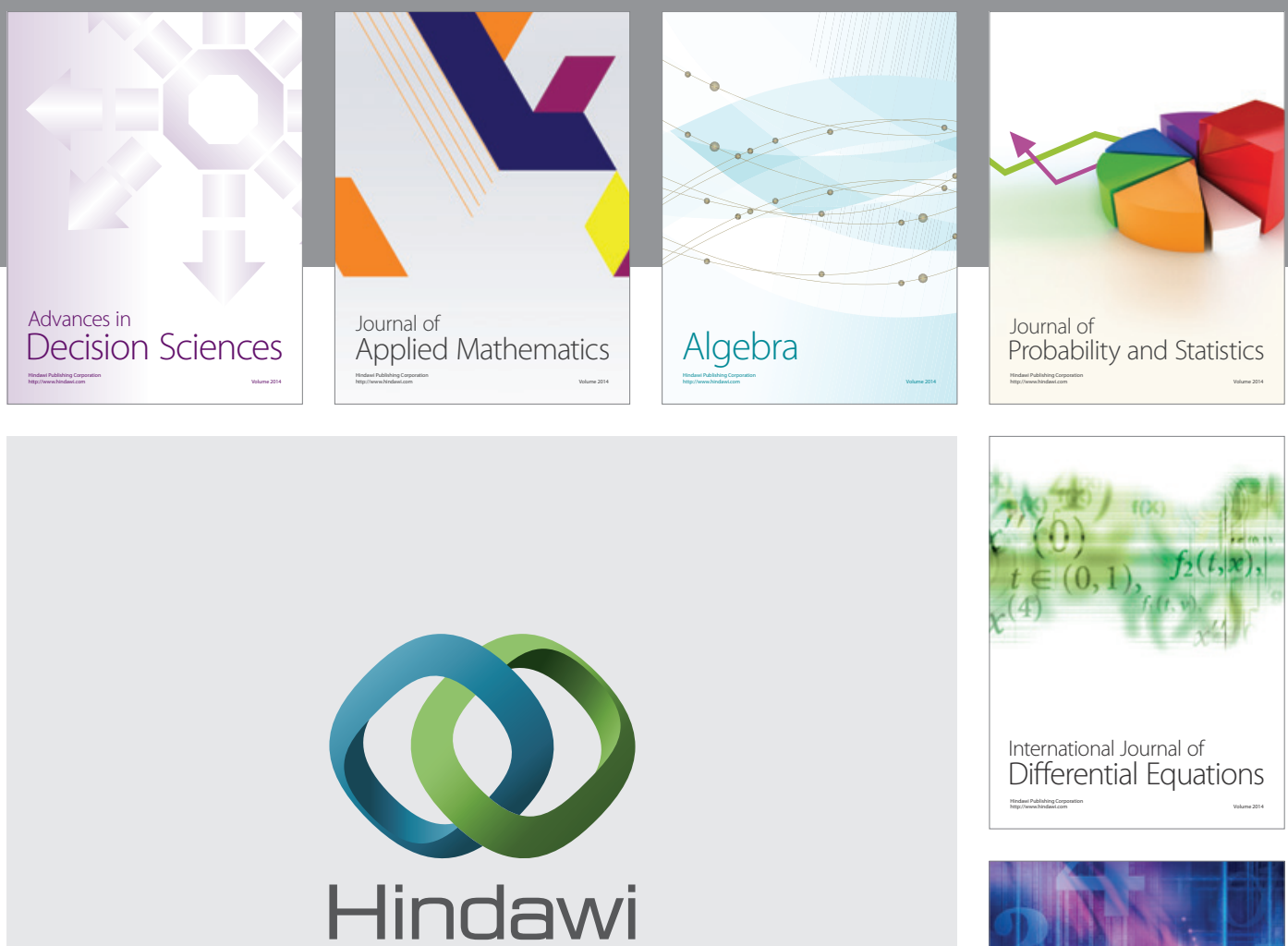

Submit your manuscripts at http://www.hindawi.com
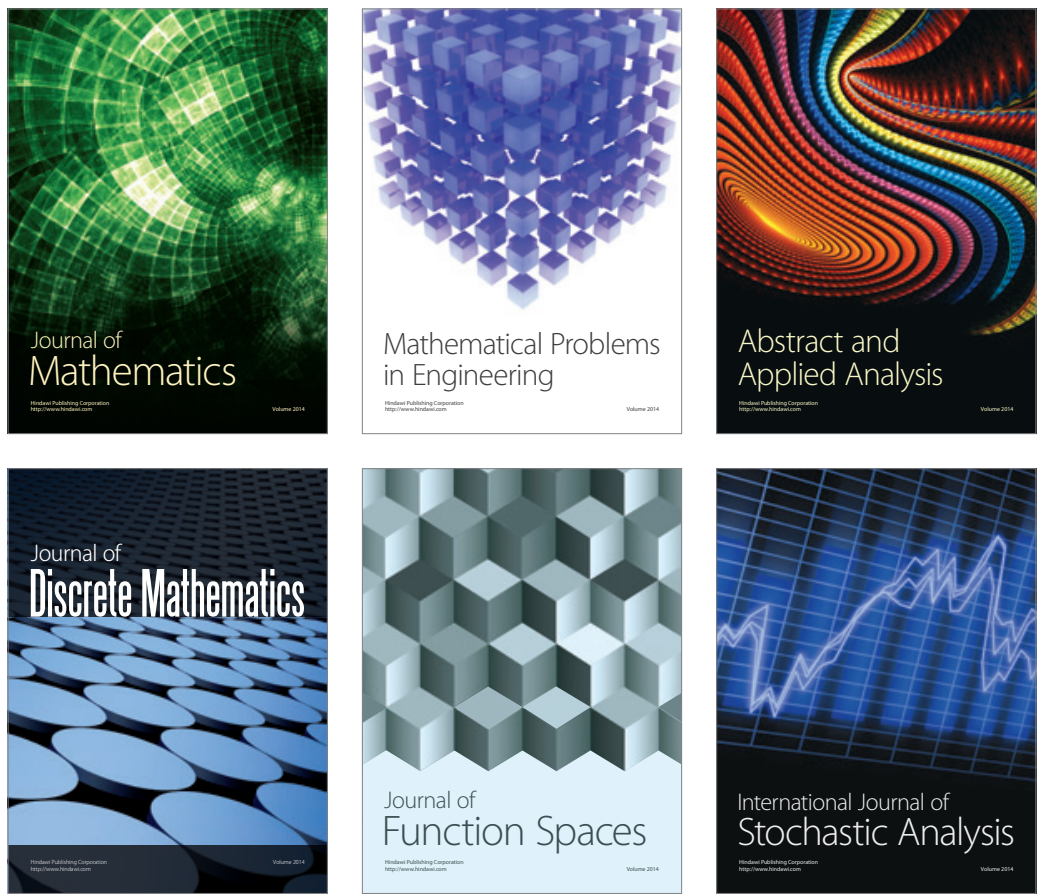

Journal of

Function Spaces

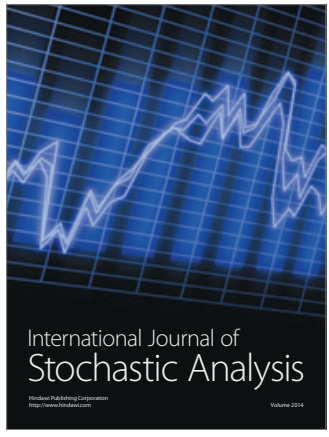

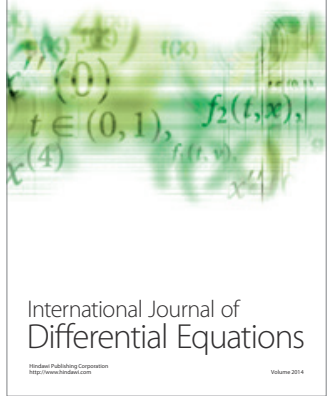
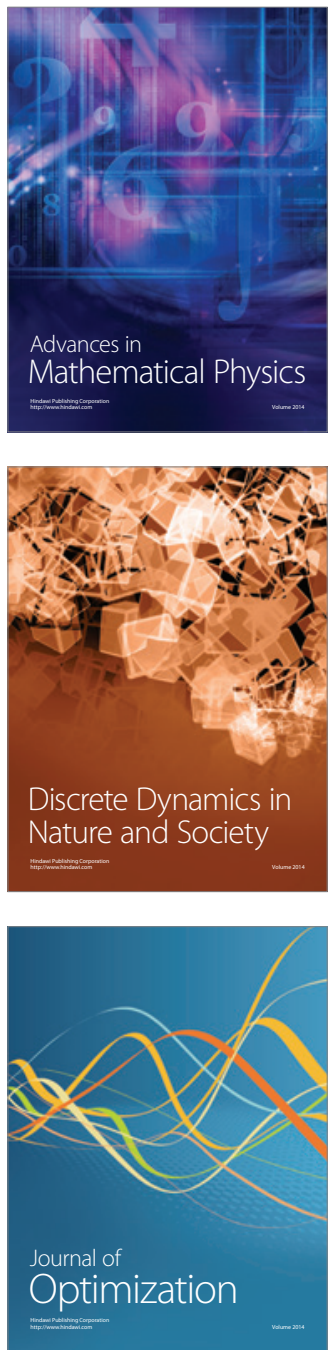